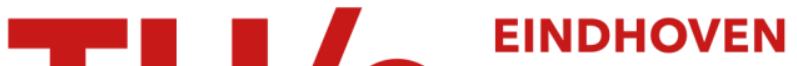 UNIVERSITY OF TECHNOLOGY
}

\section{Optimization of chaotic micromixers using finite time Lyapunov exponents}

Citation for published version (APA):

Sarkar, A., Narváez Salazar, A. E., \& Harting, J. D. R. (2012). Optimization of chaotic micromixers using finite time Lyapunov exponents. In W. Nagel, D. Kröner, \& M. Resch (Eds.), High Performance Computing in Science and Engineering '11 (pp. 325-336). Springer. https://doi.org/10.1007/978-3-642-23869-7_24

DOI:

10.1007/978-3-642-23869-7_24

Document status and date:

Published: 01/01/2012

Document Version:

Publisher's PDF, also known as Version of Record (includes final page, issue and volume numbers)

Please check the document version of this publication:

- A submitted manuscript is the version of the article upon submission and before peer-review. There can be important differences between the submitted version and the official published version of record. People interested in the research are advised to contact the author for the final version of the publication, or visit the $\mathrm{DOI}$ to the publisher's website.

- The final author version and the galley proof are versions of the publication after peer review.

- The final published version features the final layout of the paper including the volume, issue and page numbers.

Link to publication

\section{General rights}

Copyright and moral rights for the publications made accessible in the public portal are retained by the authors and/or other copyright owners and it is a condition of accessing publications that users recognise and abide by the legal requirements associated with these rights.

- Users may download and print one copy of any publication from the public portal for the purpose of private study or research.

- You may not further distribute the material or use it for any profit-making activity or commercial gain

- You may freely distribute the URL identifying the publication in the public portal.

If the publication is distributed under the terms of Article 25fa of the Dutch Copyright Act, indicated by the "Taverne" license above, please follow below link for the End User Agreement:

www.tue.nl/taverne

Take down policy

If you believe that this document breaches copyright please contact us at:

openaccess@tue.nl

providing details and we will investigate your claim. 


\title{
Optimization of Chaotic Micromixers Using Finite Time Lyapunov Exponents
}

\author{
Aniruddha Sarkar, Ariel Narváez, and Jens Harting
}

\begin{abstract}
In microfluidics mixing of different fluids is a highly non-trivial task due to the absence of turbulence. The dominant process allowing mixing at low Reynolds number is therefore diffusion, thus rendering mixing in plain channels very inefficient. Recently, passive chaotic micromixers such as the staggered herringbone mixer were developed, allowing efficient mixing of fluids by repeated stretching and folding of the fluid interfaces. The optimization of the geometrical parameters of such mixer devices is often performed by time consuming and expensive trial and error experiments. We demonstrate that the application of the lattice Boltzmann method to fluid flow in highly complex mixer geometries together with standard techniques from statistical physics and dynamical systems theory can lead to a highly efficient way to optimize micromixer geometries. The strategy applies massively parallel fluid flow simulations inside a mixer, where massless and noninteracting tracer particles are introduced. By following their trajectories we can calculate finite time Lyapunov exponents in order to quantify the degree of chaotic advection inside the mixer. The current report provides a review of our results published in [1] together with additional details on the simulation methodology.
\end{abstract}

\section{Introduction}

Microfluidics is an interdisciplinary engineering and science branch which connects physics, chemistry, biology and engineering and has applications in various scientific and industrial areas. Here, we are interested in a common building block for microfluidic systems, namely micromixers. A micromixer is a microfluidic device

Aniruddha Sarkar · Ariel Narváez · Jens Harting

Institute for Computational Physics, University of Stuttgart, Pfaffenwaldring 27, 70569 Stuttgart, Germany

Ariel Narváez · Jens Harting

Department of Applied Physics, Eindhoven University of Technology, Den Dolech 2, 5600MB

Eindhoven, The Netherlands 
used for effective mixing of different fluid constituents. A typical example is the integration as important component of chemical and biological sensors [2]. It can be used to efficiently mix for example a variety of bio-reactants such as bacteria cells, large DNA molecules, enzymes and proteins in portable integrated microsystems with minimum energy consumption. It is also used in mixing of solutions in chemical reactions [3], sequencing of nucleic acids or drug solution dilution. In recent years the demand for highly efficient and reliable micromixers has increased substantially in research and in industry. Hence, their optimized design has become an important field of research [4].

Due to the small dimensions of micromixers laminar flows are created inside the channels causing the mixing performance to be limited. Experiments on channels with complex surface topology have revealed that microscale mixing is enhanced by "chaotic advection", a process which was first reviewed by Aref in 1984 [5]. He describes how mixing is still possible even at low Reynolds number by repeated stretching and folding of fluid elements. If properly applied, this mechanism causes the interfacial area between the fluids to increase exponentially, which can then lead to an enhanced inter-material transport and thus better mixing [6].

If an external energy source is used to drive the mixing process, then the micromixer is termed as "active mixer". These external energy sources could be acoustic bubble induced vibrations, periodic variation of the flow rate, piezoelectric vibrating membranes, valves etc. The external sources are often moving components such as micropumps and they require advanced fabrication steps [7]. The second category of micromixers is based on restructuring the flow profile using static but sophisticated mixer geometries. These are termed as "passive mixer." While the fabrication of passive micromixers is generally much simpler than producing an active device, further advantages are increased reliability and the lack of any elements which generate heat. The absence of heating is an important factor for applications to biological studies where temperature is a sensitive parameter.

The mixing length and mixing time are defined as the distance and time span the fluid constituents have to flow inside the mixer in order to obtain a homogeneous mixture. An effective micromixer should reduce the mixing length and time substantially in order to achieve rapid mixing. A common practice to achieve this goal is to design passive micromixers that create alternating thin fluid lamellae. These result in an interfacial area that increases linearly with the number of lamellae rendering the diffusion process more effective and hence allowing faster mixing [8]. There are many examples of bi-lamellation [9, 10] and multi-lamellation [4], but the drawback of such devices is that the number of lamellae is generally limited due to the negative impact on the applied pressure drop caused by the microstructures inside the channel.

The drawbacks of conventional mixers based on multi-lamination techniques is overcome in the so-called "chaotic micromixer". Such a device consists of microstructured objects such as "herringbones", placed inside a microchannel. The staggered herringbone mixer (SHM) shown in Fig. 1 is the first chaotic micromixer that can be found in the literature. It was developed in 2002 by Stroock et al. [11]. The half cycles of the SHM consist of grooves with two arms which are asymmetric 

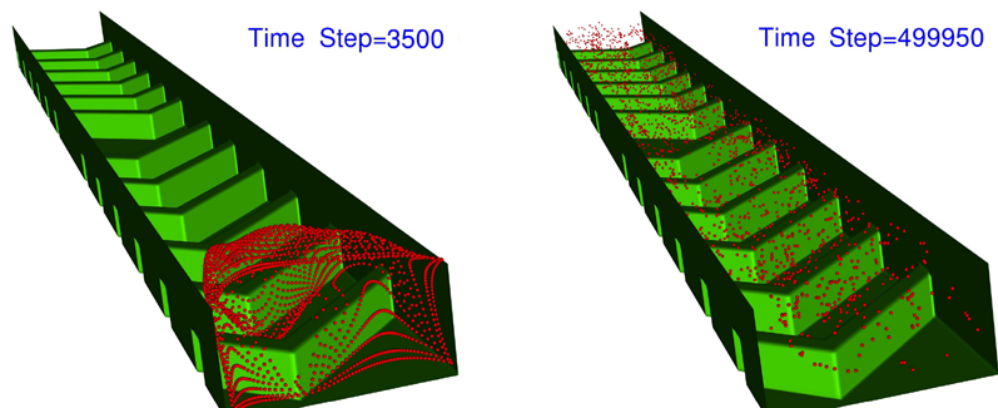

Fig. 1 A snapshot from a typical simulation of flow inside a staggered herringbone micromixer demonstrating the highly regular arrangement of tracer particles at the beginning of the simulation (left) and a fully mixed state at a late stage of the simulation (right). The fluid itself is not shown

and unequal in length. These arms are inclined at an angle of $45^{\circ}$ and the pattern interchanges every half cycle of the herringbone. The peculiar arrangement of the herringbone structure enhances the mixing process by "chaotic advection" where the interfacial area between the fluids grows exponentially in time-the most important advantage over mixers using the concept of multi-lamellation.

To compare different micromixers and to develop better ones, it is important to develop schemes to quantify their performance. Efficiency and mixing quality have been studied by various methods in the past. These include the analysis of the probability density function of the flow profiles, studying the stretching of the flow field, the Poincaré section analysis, or the intensity of segregation as introduced by Danckwerts in 1952 [6, 12]. Here, an alternative numerical optimization procedure is presented which is tailored for the optimization of chaotic micromixers and which is able to harness the power of today's high performance computers for the application to a highly practical problem. It is based on lattice Boltzmann (LB) simulations to describe the flow inside complex mixer geometries together with a measurement of finite time Lyapunov exponents (FTLE) as obtained from trajectories of massless tracer particles immersed in the flow. The Lyapunov exponent provides a quantitative measure of long term average growth rates of small initial flow perturbations and thus allows a quantification of the efficiency of chaotic transport [13, 14]. We apply Wolf's method to calculate the FTLE since the systems of interest are finite and simulations are limited to a finite time span [8]. The numerical scheme has the potential to assist an experimental optimization since geometrical parameters or fluid properties can easily be changed without requiring a new experiment. To demonstrate its applicability, the scheme is applied to evaluate the optimal parameters of the staggered herringbone mixer. Figure 1 depicts two typical snapshots from our simulations. The left figure shows a snapshot of the tracer positions just after the start of the simulations. The fluid itself is not shown. One can see that the tracer particles which are initially placed at the inflow plane of the SHM start to travel with the flow. As shown on the right hand side, towards the end of the simulation all tracer particles are homogeneously distributed throughout the mixer demonstrating that the system is fully mixed. 


\section{Simulation Method}

For a description of the fluid flow inside the micromixer, we apply the lattice Boltzmann method, a simplified approach to solve the Boltzmann equation in discrete space, time and with a limited set of discrete velocities [15]. The Boltzmann equation, given as

$$
\partial_{t} f+\mathbf{c} \cdot \nabla f=\Omega(f),
$$

describes the evolution of the velocity distribution function by molecular transport and binary intermolecular collisions. $f(\mathbf{r}, \mathbf{c}, t)$ represents the distribution of velocities in continuous position and velocity space, $\mathbf{r}$ and $\mathbf{c}$ respectively. The position $\mathbf{x}$ at which $f\left(\mathbf{x}, \mathbf{c}_{k}, t\right)$ is defined, is restricted to a discrete set of points on a regular discrete lattice with lattice constant $\Delta x$. The velocity is restricted to a set of velocities $\mathbf{c}_{k}$ implying that velocity is discretized along specific directions. $\Delta t$ denotes the discrete time step. The model we adopt is a D3Q19 model which is a 3 dimensional model with 19 different velocity directions, $k=0,1, \ldots, 18$ [16]. The right hand side of the above equation represents the collision operator which is simplified to a discretized linear Bhatnagar-Gross-Krook (BGK) form [17] that can be written as

$$
\Omega_{i}=-\omega\left(f_{k}(\mathbf{x}, t)-f_{k}^{\mathrm{eq}}(\mathbf{x}, t)\right) .
$$

Here, $\omega$ is one over the relaxation time of the system, which controls the relaxation towards the Maxwell-Boltzmann equilibrium distribution $f_{k}^{\mathrm{eq}}(\mathbf{x}, t)$. By considering small velocities and constant temperature, a discretized second order Taylor expansion of the above equilibrium distribution function can be written as

$$
f_{k}^{\mathrm{eq}}(\mathbf{x}, t)=\zeta_{k} \frac{\rho}{\rho^{\circ}}\left(1+\frac{\mathbf{c}_{k} \cdot \mathbf{u}^{\mathrm{eq}}}{c_{\mathrm{s}}{ }^{2}}+\frac{\left(\mathbf{c}_{k} \cdot \mathbf{u}^{\mathrm{eq}}\right)^{2}}{2 c_{\mathrm{s}}{ }^{4}}-\frac{\mathbf{u}^{\mathrm{eq}} \cdot \mathbf{u}^{\mathrm{eq}}}{2 c_{\mathrm{s}}{ }^{2}}\right),
$$

where $\zeta_{k}$ are the lattice weights, $\rho$ is the density, $\rho^{\circ}$ a reference density, and $c_{\mathrm{S}}=$ $(1 / \sqrt{3}) \Delta x / \Delta t$ is the speed of sound. $\mathbf{u}^{\mathrm{eq}}$ is the equilibrium velocity of the fluid, which is shifted from the mean velocity by an amount $\mathbf{g} / \omega$ under the influence of a constant acceleration $\mathbf{g}$. The evolution of the LB process takes place in two steps: the collision step where the velocities are redistributed along the directions of the lattice and the propagation step by which they are displaced along these directions. This leads to the discretized Boltzmann kinetic equation:

$$
f_{k}\left(\mathbf{x}+\Delta t \mathbf{c}_{k}, t+\Delta t\right)-f_{k}(\mathbf{x}, t)=-\omega \Delta t\left(f_{k}(\mathbf{x}, t)-f_{k}^{\mathrm{eq}}(\mathbf{x}, t)\right) .
$$

Here, the macroscopic fluid density is given by

$$
\rho(\mathbf{x}, t)=\rho^{\circ} \sum_{k} f_{k}(\mathbf{x}, t)
$$

and the macroscopic fluid velocity in the presence of external forcing is given by 


$$
\mathbf{u}(\mathbf{x}, t)=\frac{\rho^{\circ}}{\rho(\mathbf{x}, t)} \sum_{k} f_{k}(\mathbf{x}, t) \mathbf{c}_{k}-\frac{\Delta t}{2} \mathbf{g} .
$$

It can be shown by a Chapman-Enskog expansion that the macroscopic fields $\mathbf{u}$ and $\rho$ from the above equations fulfill the Navier Stokes equation in the low Mach number limit and for isothermal systems [15]. In order to simulate a fluid flow through microchannels, periodic boundary conditions are implemented along the flow direction (see Fig. 1) and no-slip bounce back boundary conditions are imposed at the channel walls.

We simulate a fluid which is hydrodynamically similar to water, flowing inside a SHM with a cross section of $96 \mu \mathrm{m} \times 192 \mu \mathrm{m}$. The length of the channel is of the order of $1536 \mu \mathrm{m}$, but can be varied in order to always accommodate a full cycle of the herringbone structure. For computational efficiency we have chosen a lattice resolution of $\Delta x=3 \mu \mathrm{m}$. Such a resolution is sufficient for a comparably simple geometry as studied in this report. However, if more sophisticated mixer geometries are to be optimized the resolution needs to be increased. Further, if mixing of multiple phases is to be simulated, periodic boundary conditions cannot be applied requiring the simulation of the full length of the mixer. In the LB method, the kinematic viscosity is related to the discrete time step through the expression $v=c_{\mathrm{s}}^{2}(\omega-\Delta t / 2)$. $\Delta t / \omega$ is chosen to be 1 and the simulated fluid has the kinematic viscosity of water, $v=10^{-6} \mathrm{~m}^{2} \mathrm{~s}-1$. This implies for the current choice of $\Delta x$ that $\Delta t=1.5 \times 10^{-6} \mathrm{~s}$ and $c_{\mathrm{S}}=1.15 \mathrm{~m} \mathrm{~s}^{-1}$. The Reynolds number $\operatorname{Re}=u L / v$ is set to the values 0.4 and 1.3, where $L=\sqrt{H^{2}+W^{2}}$ is the characteristic length of the channel. $H$ denotes the height of the channel and $W$ denotes the width of the channel.

Trajectories of massless and non-interacting tracer particles introduced into the flow are obtained by integrating the vector equation of motion

$$
\frac{\mathrm{d} \mathbf{R}_{j}}{\mathrm{~d} t}=\mathbf{u}\left(\mathbf{R}_{j}\right), \quad j=1, \ldots, P
$$

where $\mathbf{R}_{j}$ denotes the position vector of an individual tracer particle. The velocity $\mathbf{u}\left(\mathbf{R}_{j}\right)$ is obtained from the discrete LB velocity field through a trilinear interpolation scheme. After the flow simulation has reached its steady state, $P=1,000$ particles are introduced at fluid nodes in the inlet and then their velocities are integrated at each time step.

A general feature of chaotic systems is that two nearby trajectories diverge exponentially in time. The rate of divergence can be related to the strength of the flow field to create conditions for chaotic mixing. The Lyapunov exponent is a possible measure for this effect since it is related to the rate of stretching of the trajectories. It is defined by

$$
\lambda_{\infty}=\lim _{t \rightarrow \infty} \frac{1}{t} \ln \left(\frac{\mathscr{D}(t)}{\mathscr{D}(0)}\right),
$$

where $\mathscr{D}(t)$ is the distance between two trajectories at time $t . \lambda_{\infty}$ gives the value of the Lyapunov exponent as $t$ tends to infinity. Since any real system is finite it is not possible to implement this definition to quantify mixing. Also, when two trajectories 
separate from each other, this definition does not allow to understand the ongoing stretching and folding dynamics. A quantitative measure of mixing based on the Lyapunov exponent can be obtained by using the FTLE instead [18, 19]. It is defined as [20]

$$
\lambda_{\mathrm{FTLE}}=\frac{1}{\delta t} \ln \left(\frac{\mathscr{D}(t+\delta t)}{\mathscr{D}(t)}\right),
$$

where $t$ is any particular instant of time and $\delta t$ is a finite time after which the FTLE is measured. The same process is repeated over $N$ times. For large $N$ the average FTLE converges to the Lyapunov exponent [19]

$$
\lim _{N \rightarrow \infty}\left\langle\lambda_{\mathrm{FTLE}}\right\rangle_{N}=\lambda_{\infty}
$$

Wolf et al. suggested a method to calculate the FTLE from a set of experimental data $[8,21]$. Following Wolf's approach, we implement the following equation to quantify the mixer performance on the basis of the average FTLE as

$$
\langle\lambda\rangle_{N}=\frac{1}{N} \sum_{i=0}^{N-1} \frac{1}{\tau_{i}} \ln \frac{\mathscr{D}\left(t_{i}+\tau_{i}\right)}{\mathscr{D}\left(t_{i}\right)},
$$

where $t_{i}$ is the $i$ th time when a FTLE is evaluated, $\mathscr{D}\left(t_{i}+\tau_{i}\right)$ and $\mathscr{D}\left(t_{i}\right)$ are the distance at time step $t_{i}+\tau_{i}$ and $t_{i}$, respectively. $\tau_{i}$ is a multiple of $\Delta t$ and $N$ is the total number of times the particle positions are re-adjusted. If $\langle\lambda\rangle_{N}$ has a positive and non-zero value the distance between two nearby particles diverges at an exponential rate. Particle pairs which are initially very close to each other are chosen to evaluate the FTLE (i.e. with a distance $\Delta x$ ). When these particles evolve in time, the distance between them either increases or decreases. If the separation is greater than a maximum distance which is half the minimum dimension of the system $H / 2$, the distance between the particles is re-adjusted to the initial distance $\mathscr{D}\left(t_{0}\right)$ and one of the particles is placed along the line of separation in order to avoid errors due to orientation. If a replacement point cannot be found due to a surface node present at the location, a nearby fluid node is selected instead. If even such points cannot be found, the replacement is postponed to a later time step. For the implementation of the scheme, for every particle pair one of the trajectories is chosen as the fiducial path, while the position of the other particle is replaced if the distance becomes larger than the threshold value.

\section{Implementation}

During the last twelve years, our massively parallel 3D LB code (LB3D) was developed. LB3D is based on Shan and Chen's multiphase LB model [22, 23], which can be utilized to simulate a number of miscible or immiscible fluids. In addition, amphiphiles were added to the model [24]. Interactions between different fluid species are modeled by a mesoscopic force between the phases. The code was applied to a 
large number of problems to study for example the behavior of binary and ternary fluid mixtures under shear [25], the formation of surfactant mesophases [26, 27], or flow in porous media [28, 29]. Collaborations with computer scientists and software developers led to a large number of improvements to the simulation code. These include computational steering facilities, which allow the transparent access to a running simulation over the network and to change parameters or visualize output data on the fly from the user's workstation [30]. Recently, LB3D was extended to simulate typical problems arising in microfluidics including fluid flow along rough and hydrophobic surfaces [31-34]. Our group has a long standing reputation and experience in simulating suspensions using different hybrid methods consisting of an MD solver for the particle motion and various solvers for the fluid solvent [3539]. Within the last three years our lattice Boltzmann code LB3D was combined with a parallel MD code which is also the base of the results presented in this report. Within the same activity, a model for red blood cells (RBC) in plasma was implemented [38]. The cells are described as hard ellipsoids interacting with the hydrodynamic field. It was demonstrated that the code allows to describe systems containing several million RBCs on current supercomputers.

The code was ported to most available supercomputer platforms and shows a very good performance and scaling behavior. The Edinburgh parallel computing center has awarded an earlier version of LB3D with its gold medal for scaling almost linearly to 1024 processors already in 2004. Recently, improvements in the MPI communication code allowed to demonstrate strong scaling for up to 262144 cores on the BlueGene/P system Jugene in Jülich [40, 41]. The code was the main application within the TeraGyroid project where it was used on a prototype computational grid consisting of all national supercomputers in the UK and a number of machines in the US and received various prizes. See Fig. 2 for a comparison of the performance on the XC2 (Karlsruhe), HECToR (Edinburgh), Huygens (Amsterdam) and Juropa (Jülich). It is interesting to note that even though the XC2 is

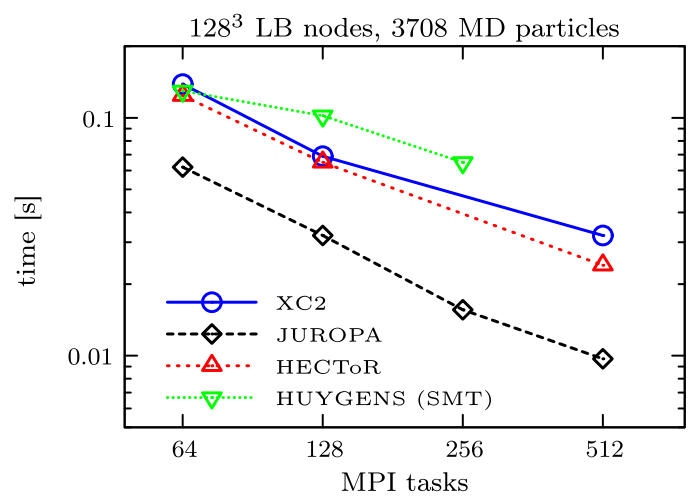

Fig. 2 Scaling and performance comparison of LB3D on the XC2 at SSC Karlsruhe, HECToR at EPCC, Huygens at SARA, and JUROPA at JSC. The studied system is comparably small which is the reason for the less good scaling compared to what we generally observe 
the oldest available platform it still performs reasonably well and has proven as a reliable workhorse during the last years. The scaling behavior in the presented plot is not very good on larger core counts because of the too small system used for the benchmark.

\section{Results}

In this section we present how FTLE can be utilized for an optimization strategy for chaotic micromixers. As an example, the influence of different parameters which directly affect the performance of the SHM is evaluated. These are the ratio of height of the grooves to the height of the channel $\alpha$, the ratio of the horizontal length of the long arm to the channel width $\beta$, the ratio of distance between the grooves to the length of the channel $\gamma$ and the number of grooves per half cycle $n$. While keeping all other parameters fixed, the width fraction $(\beta)$ is varied within the range of 0.22 and 0.82 and the distance fraction $(\gamma)$ from 0.04 to 0.11 . The width of the grooves is kept fixed at $24 \mu \mathrm{m}$ for all simulations. Then, the number of grooves per half cycle $(n)$ is varied from 2 to 10 and the height fraction $(\alpha)$ from 0.125 to 0.343 . One has to take care of a thorough convergence of the simulations since $\langle\lambda\rangle_{N}$ fluctuates before finally converging to a particular value after $\sim 6.0 \times 10^{5}$ time steps. Therefore, simulations are run until the FTLE have thoroughly converged before evaluating the tracer trajectories. The effect of the geometry can be measured by comparing the average of the converged FTLE which is denoted by $\lambda$. The error bars in Figs. 3 to 4 are given by the standard deviation of the data from the point where it has converged. A possible reduction of the required computing time can be achieved by stopping the lattice Boltzmann simulation when a steady state flow field has been obtained since the computational effort for the tracer particles is small compared to time
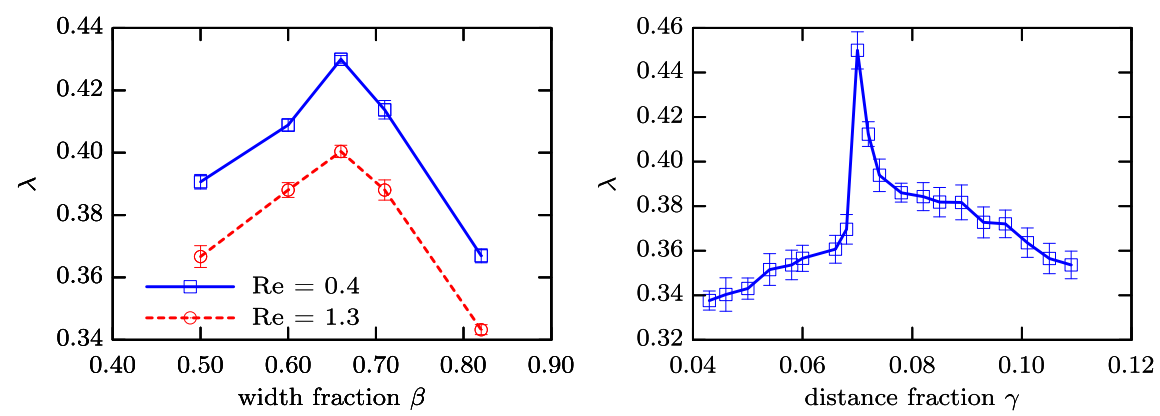

Fig. 3 Left: A maximum of the variation of the maximum averaged finite time Lyapunov exponent $\lambda$ with different width fraction $\beta$ can be obtained for a width fraction of $\beta=2 / 3$. While the position of the maximum is not affected by changing Re, the absolute values change. Right: The FTLE rises with the increase of the distance fraction $\gamma$ until it reaches a distinct peak. Then, the curve decreases demonstrating an optimized performance of the mixer at $\gamma=0.07$ [1] 

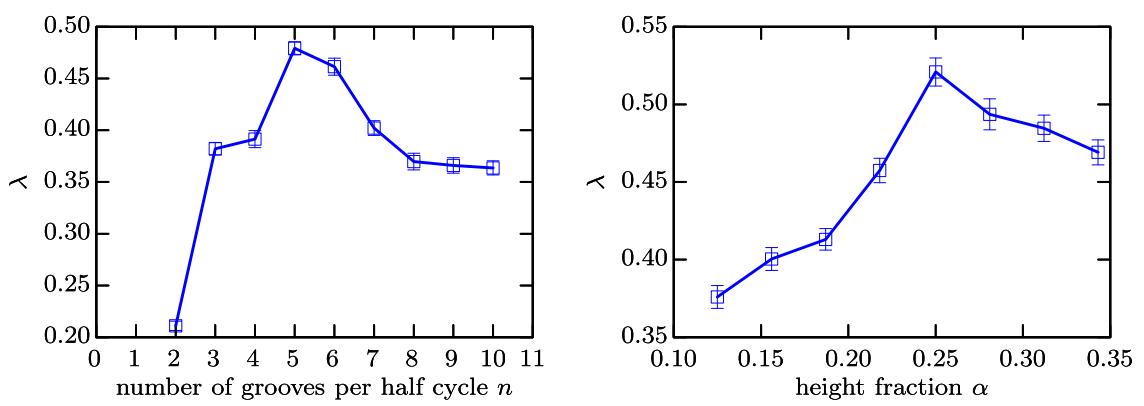

Fig. 4 Left: The variation of $\lambda$ with the number of grooves per half cycle $(n)$ shows that the SHM with $n=5$ performs best. Right: A variation of the height fraction $(\alpha)$ indicates that the maximum FTLE can be obtained for $\alpha=0.25$ [1]

required for the fluid solver. However, for complex geometries and chaotic flows several hundred thousand timesteps can be required to obtain the steady state and a thorough convergence study would be needed for every different channel geometry.

The left part of Fig. 3 depicts the variance of $\lambda$ and as such the performance of the SHM with respect to $\beta$ for two different Reynolds numbers, $\operatorname{Re}=0.4$ and 1.3 . Due to the symmetry of the mixer geometry, only values for $\beta \geq 0.5$ are plotted. The datasets peak at $\beta=2 / 3$ implying that the degree of chaotic advection is maximized for this particular value of the width fraction $\beta$. The measurements at different Reynolds numbers depict that changing the driving force does change the absolute value of $\lambda$, but has no influence on the general shape of the curve. This is confirmed by similar studies of the Re dependence for other geometrical parameters and various different driving forces. Therefore, we restrict ourselves to $\operatorname{Re}=1.3$ for all further simulations. Our findings are consistent with the original experimental work of Stroock et al. [11] as well as numerical optimizations by Stroock and McGraw [42]. Both publications show that $\beta=2 / 3$ generates a maximum swirling motion of the fluid trajectories. However such analysis with dyes or concentration profiles does not allow to obtain an insight into the behavior of the flow field, while the FTLE does.

In the right inset of Fig. 3 data from a set of simulations with $\beta$ fixed at the optimized value of $2 / 3$ and the distance fraction $\gamma$ being varied from 0.04 to 0.11 is shown. It can be observed that after a moderate increase of $\lambda$ with $\gamma$, the curve has a sharp peak at $\gamma=0.07$, which corresponds to a value of $d=105 \mu \mathrm{m}$ for the current choice of $\Delta x$. Afterwards, $\lambda$ decreases in a similar fashion as for small $\gamma$, but still at higher absolute values.

In the following the number of grooves per half-cycle $n$ is varied from 2 to 10 . It can be understood from the left inset of Fig. 4 that a variation of $n$ has the largest impact on the performance of the mixer as compared to $\beta$ or $\gamma$. For the current setup, by variation of $n$ it is possible to change the value of $\lambda$ by a factor of 2.3 as compared to 1.2 for $\beta$ and 1.3 for $\gamma$. The data clearly demonstrates that a staggered herringbone mixer with $n=5$ performs best. Similar to our work, Li and Chen performed LB simulations and used tracers to follow the flow field [43]. They, however, quantify 
mixing by computing the standard deviation of the local tracer concentration and conclude that mixers with $n=5$ or $n=6$ perform best. This result is in agreement with our finding, but the FTLE analysis clearly shows that the channel with $n=5$ performs better than the one with $n=6$.

The final parameter to be considered is the ratio of the half depth of the grooves to the height of the channel $\alpha$. Figure 4 (right) depicts the average value of the converged Lyapunov exponents for $\alpha$ between 0.125 and 0.343 . After a strong increase of the curve the data has a maximum at $\alpha=0.25$. For larger $\alpha$ the value of $\lambda$ decreases again. Our result are confirmed by the original experimental analysis of Stroock et al. [11].

\section{Summary}

Passive chaotic micromixers can be successfully applied to improve mixing at the microscale where turbulence is absent and only diffusion can be used for mixing. These mixers provide a large fluid-fluid interface by repeatedly stretching and folding of these interfaces. The performance of such mixers depends on the rate at which "chaotic advection" of the fluid takes place. In this work we have demonstrated an efficient numerical scheme which allows the quantification of "chaotic advection" and thus the performance of a micromixer. The scheme is based on our well developed massively parallel LB solver LB3D to describe the time dependent flow field in complex mixer geometries combined with Wolf's method to compute FTLE from passive tracer trajectories. We have utilized the XC2 in Karlsruhe to demonstrate the applicability of the quantification method by applying it to optimize the geometry of the staggered herringbone mixer. By performing a systematic variation of the relevant geometrical parameters we obtained a set of optimal values $\alpha=0.25$, $\beta=2 / 3, \gamma=0.07$ and $n=5$ which is consistent with literature data published by others. An important feature of the method presented here is that it allows optimization of the mixing performance by direct investigation of the underlying dynamical process [1]. Currently we are extending our method to make use of the multiphase and multicomponent capabilities of our lattice Boltzmann implementation in order to study the mixing of multiphase flows in microchannels. This is of course a more realistic scenario which has received surprisingly little attention in the literature so far. A possible explanation for the small number of publications on this topic is the complicated interaction between the process of chaotic advection together with the parameters determining the diffusion between different fluid species.

Acknowledgments. The authors thank F. Janoschek, F. Raischel, G.J.F. van Heijst, and M. Pattantyús-Ábrahám for fruitful discussions. This work was financed within the DFG priority program "nano- and microfluidics", the DFG collaborative research center 716, and by the NWO/STW VIDI grant of J. Harting. A. Narváez thanks Deutscher Akademischer Austauschdienst (DAAD) for financial support. We thank the Scientific Supercomputing Center Karlsruhe for providing the computing time and technical support for the presented work. 


\section{References}

1. A. Sarkar, A. Narváez, and J. Harting. Quantification of the degree of mixing in chaotic micromixers using finite time Lyapunov exponents. Submitted for publication, arXiv:1012.5549, 2010.

2. M. A. Burns. Microfabricated structures for integrated DNA analysis. Proc. National Acad. Sci. USA, 68(93):5556-5561, 1996.

3. P. Watts and S. Haswell. Microfluidic combinatorial chemistry. Curr. Opin. Chem. Biol., 7:380-387, 1996.

4. V. Hessel, H. Loewe, and F. Schoenfeld. Micromixers-A review on active and passive mixing principles. Chem. Eng. Sci., 60:2479-2501, 2005.

5. H. Aref. Stirring by chaotic advection. J. Fluid Mech., 143:1-21, 1984.

6. H. Kim and A. Beskok. Quantification of chaotic strength and mixing in a micro fluidic system. J. Micromech. Microeng., 17:2197-2210, 2007.

7. C. Zhang, D. Xing, and Y. Li. Micropumps, microvalves and micromixers within pcr microfluidic chips: Advances and trends. Biotech. Advances, 25:483-514, 2007.

8. F. G. Bessoth, A. de Mello, and A. Manz. Microstructure for efficient continuous flow mixing. Analyt. Comm., 36:213-215, 1999.

9. D. Gobby, P. Angeli, and A. Gavriliidis. Mixing characteristics of a T-type microfluidic mixers. J. Micromech. Microeng., 11:126-132, 2001.

10. Y. Mingquiang and $\mathrm{H}$. Bau. The kinematics of bend-induced stirring in micro-conduits. In Proc. ASME Intl. Mech. Sys. (MEMS'97), Nagoya, Japan, pp. 96-101, 2000.

11. A. Strook, S. Dertinger, A. Adjari, I. Mezic, H. Stone, and G. Whiteside. Chaotic mixer for microchannels. Science, 295:647-651, 2002.

12. T. K. Kang, M. K. Singh, T. H. Kwon, and P. D. Anderson. Chaotic mixing using periodic and aperiodic sequences of mixing protocols. Microfluids and Nanofluids, 4(6):589-599, 2007.

13. C. Ziemann, L. A. Smith, and J. Kurths. Localized Lyapunov exponents and the prediction of predictability. Phys. Lett. A, 4:237-251, 2000.

14. G. Lapeyre. Characterization of finite-time Lyapunov exponents and vectors in twodimensional turbulence. Chaos, 12(3):688-698, 2002.

15. S. Succi. The Lattice Boltzmann Equation for Fluid Dynamics and Beyond. Oxford University Press, London, 2001.

16. Y. H. Qian, D. d'Humieres, and P. Lallemand. Lattice BGK models for Navier-Stokes equation. Europhys. Lett., 17(6):479-484, 1992.

17. P. Bhatnagar, E. Gross, and M. Krook. A model for collision process in gases. Small amplitude process in charged and neutral one-component systems. Phys. Rev., 94:511-525, 1954.

18. D. Ruiquiang and L. Jianping. Nonlinear finite-time Lyapunov exponent and predictability. Phys. Lett. A, 364:396-400, 2007.

19. X. Tang and A. Boozer. Finite time Lyapunov exponent and chaotic advection-diffusion equation. Physica D, 95:283-305, 1996.

20. Y. Lee, C. Shih, P. Tabeling, and C.-M. Ho. Experimental study and non-linear dynamics of time-periodic micro chaotic mixers. J. Fluid Mech., 575:425-448, 2007.

21. A. Wolf, J. B. Swift, H. L. Swinney, and J. A. Vastano. Determining Lyapunov exponents from a time series. Physica D, 16:285-317, 1985.

22. X. Shan and H. Chen. Lattice Boltzmann model for simulating flows with multiple phases and components. Phys. Rev. E, 47(3):1815, 1993.

23. X. Shan and H. Chen. Simulation of nonideal gases and liquid-gas phase transitions by the lattice Boltzmann equation. Phys. Rev. E, 49(4):2941, 1994.

24. H. Chen, B. M. Boghosian, P. V. Coveney, and M. Nekovee. A ternary lattice Boltzmann model for amphiphilic fluids. Proc. R. Soc. Lond. A, 456:2043, 2000.

25. J. Harting, M. Harvey, J. Chin, M. Venturoli, and P. V. Coveney. Large-scale lattice Boltzmann simulations of complex fluids: Advances through the advent of computational grids. Phil. Trans. R. Soc. Lond. A, 363:1895-1915, 2005. 
26. G. Giupponi, J. Harting, and P. V. Coveney. Emergence of rheological properties in lattice Boltzmann simulations of gyroid mesophases. Europhys. Lett., 73:533-539, 2006.

27. N. González-Segredo, J. Harting, G. Giupponi, and P. V. Coveney. Stress response and structural transitions in sheared gyroidal and lamellar amphiphilic mesophases: Lattice-Boltzmann simulations. Phys. Rev. E, 73:031503, 2006.

28. A. Narváez, T. Zauner, F. Raischel, R. Hilfer, and J. Harting. Quantitative analysis of numerical estimates for the permeability of porous media from lattice-Boltzmann simulations. J. Stat. Mech: Theor. Exp., 2010:P211026, 2010.

29. A. Narváez and J. Harting. A D3Q19 lattice-Boltzmann pore-list code with pressure boundary conditions for permeability calculations. Advances in Applied Mathematics and Mechanics, 2:685, 2010.

30. J. Chin, J. Harting, S. Jha, P. V. Coveney, A. R. Porter, and S. M. Pickles. Steering in computational science: Mesoscale modelling and simulation. Contemporary Physics, 44(5):417-434, 2003.

31. J. Harting, C. Kunert, and H. Herrmann. Lattice Boltzmann simulations of apparent slip in hydrophobic microchannels. Europhys. Lett., 75:328-334, 2006.

32. C. Kunert and J. Harting. Roughness induced apparent boundary slip in microchannel flows. Phys. Rev. Lett., 99:176001, 2007.

33. J. Hyväluoma and J. Harting. Slip flow over structured surfaces with entrapped microbubbles. Phys. Rev. Lett., 100:246001, 2008.

34. C. Kunert, J. Harting, and O. I. Vinogradova. Random-roughness hydrodynamic boundary conditions. Phys. Rev. Lett., 105:016001, 2010.

35. A. Komnik, J. Harting, and H. J. Herrmann. Transport phenomena and structuring in shear flow of suspensions near solid walls. J. Stat. Mech: Theor. Exp., P12003, 2004.

36. M. Hecht, J. Harting, T. Ihle, and H. J. Herrmann. Simulation of claylike colloids. Phys. Rev. E, 72:011408, 2005.

37. J. Harting, H. J. Herrmann, and E. Ben-Naim. Anomalous distribution functions in sheared suspensions. Europhys. Lett., 83:30001, 2008.

38. F. Janoschek, F. Toschi, and J. Harting. Simplified particulate model for coarse-grained hemodynamics simulations. Phys. Rev. E, 82:056710, 2010.

39. F. Jansen and J. Harting. From Bijels to Pickering emulsions: A lattice Boltzmann study. Phys. Rev. E, 83:046707, 2011.

40. D. Groen, O. Henrich, F. Janoschek, P. Coveney, and J. Harting. Lattice-Boltzmann methods in fluid dynamics: Turbulence and complex colloidal fluids. In W. F. Bernd Mohr, editor, Jülich Blue Gene/P Extreme Scaling Workshop 2011. Jülich Supercomputing Centre, 52425 Jülich, Germany, apr 2011. FZJ-JSC-IB-2011-02; http://www2.fz-juelich.de/jsc/docs/autoren2011/ mohr1/.

41. J. Harting, F. Jansen, S. Frijters, F. Janoschek, and F. Günther. Nanoparticles as emulsion stabilizers. inSiDE, 9(1):48, 2011.

42. A. D. Stroock and G. J. McGraw. Investigation of the staggered herringbone mixer with a simple analytical model. Phil. Trans. R. Soc. Lond. A, 362:923-935, 2004.

43. C. Li and T. Chen. Simulation and optimization of chaotic micromixer using lattice Boltzmann method. Sensors and Actuators B, 106:871-877, 2005. 